ANALYSIS \& PDE Volume $9 \quad$ No. $3 \quad 2016$

(1)

MICHAEL BENNETT, ALEXANDER IOSEVICH AND KRYSTAL TAYLOR

FINITE CHAINS INSIDE THIN SUBSETS OF $\mathbb{R}^{d}$ 


\title{
FINITE CHAINS INSIDE THIN SUBSETS OF $\mathbb{R}^{d}$
}

\author{
Michael Bennett, Alexander Iosevich and Krystal Taylor
}

\begin{abstract}
In a recent paper, Chan, Łaba, and Pramanik investigated geometric configurations inside thin subsets of Euclidean space possessing measures with Fourier decay properties. In this paper we ask which configurations can be found inside thin sets of a given Hausdorff dimension without any additional assumptions on the structure. We prove that if the Hausdorff dimension of $E \subset \mathbb{R}^{d}, d \geq 2$, is greater than $\frac{1}{2}(d+1)$ then, for each $k \in \mathbb{Z}^{+}$, there exists a nonempty interval $I$ such that, given any sequence $\left\{t_{1}, t_{2}, \ldots, t_{k}: t_{j} \in I\right\}$, there exists a sequence of distinct points $\left\{x^{j}\right\}_{j=1}^{k+1}$ such that $x^{j} \in E$ and $\left|x^{i+1}-x^{i}\right|=t_{j}$ for $1 \leq i \leq k$. In other words, $E$ contains vertices of a chain of arbitrary length with prescribed gaps.
\end{abstract}

\section{Introduction}

The problem of determining which geometric configurations one can find inside various subsets of Euclidean space is a classical subject. The basic problem is to understand how large a subset of Euclidean space must be to be sure that it contains the vertices of a congruent and possibly scaled copy of a given polyhedron or another geometric shape. In the case of a finite set, "large" refers to the number of points, while in infinite sets it refers to the Hausdorff dimension or Lebesgue density. The resulting class of problems has been attacked by a variety of authors using combinatorial, number theoretic, ergodic, and Fourier analytic techniques, creating a rich set of ideas and interactions.

We begin with a comprehensive result due to Tamar Ziegler [2006], which generalizes an earlier result due to Furstenberg, Katznelson and Weiss [Furstenberg et al. 1990]. See also [Bourgain 1986].

Theorem 1.1 [Ziegler 2006]. Let $E \subset \mathbb{R}^{d}$ be of positive upper Lebesgue density, in the sense that

$$
\limsup _{R \rightarrow \infty} \frac{\mathscr{L}^{d}\left\{E \cap[-R, R]^{d}\right\}}{(2 R)^{d}}>0,
$$

where $\mathscr{L}^{d}$ denotes the d-dimensional Lebesgue measure. Let $E_{\delta}$ denote the $\delta$-neighborhood of E. Let $V=\left\{\mathbf{0}, v^{1}, v^{2}, \ldots, v^{k-1}\right\} \subset \mathbb{R}^{d}$, where $k \geq 2$ is a positive integer. Then there exists $l_{0}>0$ such that, for any $l>l_{0}$ and any $\delta>0$, there exists $\left\{x^{1}, \ldots, x^{k}\right\} \subset E_{\delta}$ congruent to $l V=\left\{\mathbf{0}, l v^{1}, \ldots, l v^{k-1}\right\}$.

In particular, this result shows that we can recover every simplex similarity type and sufficiently large scaling inside a subset of $\mathbb{R}^{d}$ of positive upper Lebesgue density. It is reasonable to wonder whether the assumptions of Theorem 1.1 can be weakened, but the following result, due to Maga [2010], shows that

This work was partially supported by the NSF Grant DMS10-4504 and the NSA Grant H98230-15-1-0319.

MSC2010: primary 28A75, 42B10; secondary 53C10.

Keywords: classical analysis and ODEs, combinatorics, metric geometry, chains, geometric measure theory, geometric configurations, Hausdorff dimension, Falconer distance problem. 
the conclusion may fail even if we replace the upper Lebesgue density condition with the assumption that the set is of dimension $d$.

Theorem 1.2 [Maga 2010]. For any $d \geq 2$ there exists a full-dimensional compact set $A \subset \mathbb{R}^{d}$ such that A does not contain the vertices of any parallelogram. If $d=2$ then, given any triple of points $x^{1}, x^{2}, x^{3}$, $x^{j} \in A$, there exists a full-dimensional compact set $A \subset \mathbb{R}^{2}$ such that $A$ does not contain the vertices of any triangle similar to $\triangle x^{1} x^{2} x^{3}$.

In view of Maga's result, it is reasonable to ask whether interesting point configurations can be found inside thin sets under additional structural hypotheses. This question was recently addressed by Chan, Łaba, and Pramanik [Chan et al. 2013]. Before stating their result, we provide two relevant definitions.

Definition 1.3. Fix integers $n \geq 2, p \geq 3$ and $m=n\left\lceil\frac{1}{2}(p+1)\right\rceil$. Suppose $B_{1}, \ldots, B_{p}$ are $n \times(m-n)$ matrices.

(a) We say that $E$ contains a $p$-point $\mathscr{B}$-configuration if there exist vectors $z \in \mathbb{R}^{n}$ and $w \in \mathbb{R}^{m-n} \backslash \overrightarrow{0}$ such that

$$
\left\{z+B_{j} w\right\}_{j=1}^{p} \subset E .
$$

(b) Moreover, given any finite collection of subspaces $V_{1}, \ldots, V_{q} \subset \mathbb{R}^{m-n}$ with $\operatorname{dim}\left(V_{i}\right)<m-n$, we say that $E$ contains a nontrivial $p$-point $\mathscr{B}$-configuration with respect to $\left(V_{1}, \ldots, V_{q}\right)$ if there exist vectors $z \in \mathbb{R}^{n}$ and $w \in \mathbb{R}^{m-n} \backslash \bigcup_{i=1}^{q} V_{i}$ such that

$$
\left\{z+B_{j} w\right\}_{j=1}^{p} \subset E .
$$

Definition 1.4. Fix integers $n \geq 2, p \geq 3$ and $m=n\left\lceil\frac{1}{2}(p+1)\right\rceil$. We say that a set of $n \times(m-n)$ matrices $\left\{B_{1}, \ldots, B_{p}\right\}$ is nondegenerate if

$$
\operatorname{rank}\left(\begin{array}{c}
B_{i_{2}}-B_{i_{1}} \\
\vdots \\
B_{i_{m / n}}-B_{i_{1}}
\end{array}\right)=m-n
$$

for any distinct indices $i_{1}, \ldots, i_{m / n} \in\{1, \ldots, p\}$.

Theorem 1.5 [Chan et al. 2013]. Fix integers $n \geq 2, p \geq 3$ and $m=n\left\lceil\frac{1}{2}(p+1)\right\rceil$. Let $\left\{B_{1}, \ldots, B_{p}\right\}$ be a collection of $n \times(m-n)$ nondegenerate matrices in the sense of Definition 1.4. Then, for any constant $C$, there exists a positive number $\epsilon_{0}=\epsilon_{0}\left(C, n, p, B_{1}, \ldots, B_{p}\right) \ll 1$ with the following property: Suppose the set $E \subset \mathbb{R}^{n}$ with $|E|=0$ supports a positive, finite Radon measure $\mu$ with two conditions:

(a) Ball condition: $\sup _{x \in E, 0<r<1} \mu(B(x, r)) / r^{\alpha} \leq C$ if $n-\epsilon_{0}<\alpha<n$.

(b) Fourier decay: $\sup _{\xi \in \mathbb{R}^{n}}|\hat{\mu}(\xi)|(1+|\xi|)^{\beta / 2} \leq C$.

\section{Then:}

(i) E contains a p-point $\mathscr{B}$-configuration in the sense of Definition 1.3(a).

(ii) Moreover, for any finite collection of subspaces $V_{1}, \ldots, V_{q} \subset \mathbb{R}^{m-n}$ with $\operatorname{dim}\left(V_{i}\right)<m-n$, E contains a nontrivial p-point $\mathscr{B}$-configuration with respect to $\left(V_{1}, \ldots, V_{q}\right)$ in the sense of Definition $1.3(b)$. 


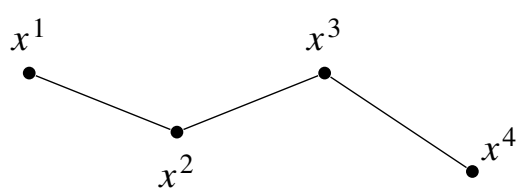

Figure 1. A 3-chain.

One can check that the Chan-Łaba-Pramanik result covers some geometric configurations but not others. For example, their nondegeneracy condition allows them to consider triangles in the plane, but not simplexes in $\mathbb{R}^{3}$ where three faces meet at one of the vertices at right angles, forming a three-dimensional corner. Most relevant to this paper is the fact that the conditions under which Theorem 1.5 holds are satisfied for chains (see Definition 1.6 below), but the conclusion requires decay properties for the Fourier transform of a measure supported on the underlying set. We shall see that, in the case of chains, such an assumption is not needed and the existence of a wide variety of chains can be established under an explicit dimensional condition alone.

Focus of this article. We establish that a set of sufficiently large Hausdorff dimension, with no additional assumptions, contains an arbitrarily long chain with vertices in the set and preassigned admissible gaps.

Definition 1.6 (see Figure 1). A $k$-chain in $E \subset \mathbb{R}^{d}$ with gaps $\left\{t_{i}\right\}_{i=1}^{k}$ is a sequence

$$
\left\{x^{1}, x^{2}, \ldots, x^{k+1}: x^{j} \in E,\left|x^{i+1}-x^{i}\right|=t_{i}, 1 \leq i \leq k\right\} .
$$

We say that the chain is nondegenerate if all the $x^{j}$ are distinct.

Our main result is the following:

Theorem 1.7. Suppose that the Hausdorff dimension of a compact set $E \subset \mathbb{R}^{d}, d \geq 2$, is greater than $\frac{1}{2}(d+1)$. Then, for any $k \geq 1$, there exists an open interval $\tilde{I}$ such that for any $\left\{t_{i}\right\}_{i=1}^{k} \subset \tilde{I}$ there exists a nondegenerate $k$-chain in $E$ with gaps $\left\{t_{i}\right\}_{i=1}^{k}$.

In the course of establishing Theorem 1.7 we shall prove the following result, which is interesting in its own right and has a number of consequences for Falconer-type problems. See [Falconer 1985; Erdog̃an 2005; Wolff 1999] for the background and the latest results pertaining to the Falconer distance problem.

Theorem 1.8. Suppose that $\mu$ is a compactly supported, nonnegative Borel measure such that

$$
\mu(B(x, r)) \leq C r^{s_{\mu}}
$$

for some $s_{\mu} \in\left(\frac{1}{2}(d+1), d\right]$, where $B(x, r)$ is the ball of radius $r>0$ centered at $x \in \mathbb{R}^{d}$. Then, for any $t_{1}, \ldots, t_{k}>0$ and $\epsilon>0$,

$$
\mu \times \mu \times \cdots \times \mu\left\{\left(x^{1}, x^{2}, \ldots, x^{k+1}\right): t_{i}-\epsilon \leq\left|x^{i+1}-x^{i}\right| \leq t_{i}+\epsilon, i=1,2, \ldots, k\right\} \leq C \epsilon^{k} .
$$

Corollary 1.9. Given a compact set $E \subset \mathbb{R}^{d}, d \geq 2, k \geq 1$, define

$$
\Delta_{k}(E)=\left\{\left|x^{1}-x^{2}\right|,\left|x^{2}-x^{3}\right|, \ldots,\left|x^{k}-x^{k+1}\right|: x^{j} \in E\right\} .
$$


Suppose that the Hausdorff dimension of $E$ is greater than $\frac{1}{2}(d+1)$. Then

$$
\mathscr{L}^{k}\left(\Delta_{k}(E)\right)>0 .
$$

Remark 1.10. Suppose that $E \subset \mathbb{R}^{d}$ has Hausdorff dimension $s>\frac{1}{2}(d+1)$ and is Ahlfors-David regular, i.e., there exists $C>0$ such that, for every $x \in E$,

$$
C^{-1} r^{s} \leq \mu(B(x, r)) \leq C r^{s}
$$

(where $\mu$ is the restriction of the $s$-dimensional Hausdorff measure to $E$ ). Then, using the techniques in [Eswarathasan et al. 2011] along with Theorem 1.8, one can show that, for any sequence of positive real numbers $t_{1}, t_{2}, \ldots, t_{k}$, the upper Minkowski dimension of

$$
\left\{\left(x^{1}, x^{2}, \ldots, x^{k+1}\right) \in E^{k+1}:\left|x^{j+1}-x^{j}\right|=t_{j}, 1 \leq j \leq k\right\}
$$

does not exceed $(k+1) \operatorname{dim}_{\mathscr{H}}(E)-k$.

\section{Proof of Theorem 1.7 and Theorem 1.8}

The strategy for this section is as follows:

We begin by dividing both sides of (1-2) by $\epsilon^{k}$. The left side becomes

$$
\epsilon^{-k} \mu \times \cdots \times \mu\left\{\left(x^{1}, \ldots, x^{k+1}\right): t_{i}-\epsilon \leq\left|x^{i+1}-x^{i}\right| \leq t_{i}+\epsilon, i=1,2, \ldots, k\right\},
$$

which can be interpreted as the density of $\epsilon$-approximate chains in $E \times \cdots \times E$.

Theorem 1.8 gives an upper bound on this expression that is independent of $\epsilon$. This is accomplished using an inductive argument on the chain length coupled with repeated application of an earlier result from [Iosevich et al. 2014], in which the authors establish $L^{2}(\mu)$ mapping properties of certain convolution operators. This upper bound is important in the final section, where we define a measure on the set of chains.

Next, we acquire a lower bound on (2-1). This result was already established in the case $k=1$ in [Iosevich et al. 2012], where the authors show that the density of $\epsilon$-approximate 1-chains with gap size $t$ is bounded below, independent of $\epsilon$, for all $t$ in a nonempty open interval $I$. Using a pigeonholing argument, we extend the result in [Iosevich et al. 2012] to obtain a lower bound on (2-1) in the case that every gap is of equal size $t$ for some $t \in I$. To obtain a lower bound on chains with variable gap size, we show that the density of $\epsilon$-approximate $k$-chains is continuous as a function of gap sizes. Furthermore, we use the lower bound on chains with constant gaps to prove that this continuous function is not identically zero. We conclude that the density of $\epsilon$-approximate $k$-chains is bounded below, independent of $\epsilon$ and independent of the gap sizes, as long as all gap sizes fall within some interval $\tilde{I}$ around $t$.

In the final section, we address the issue of nondegeneracy. To this end, we reinterpret the density of $\epsilon$-approximate $k$-chains as a measure supported in $E^{k+1}$ and show that it converges to a new measure, $\Lambda_{\vec{t}}^{k}$, as $\epsilon \downarrow 0$. This new measure is shown to be supported on "exact" $k$-chains $(\epsilon=0)$ with admissible gaps. We next show that the measure of the set of degenerate chains is 0 , and we conclude that the mass of $\Lambda_{\vec{t}}^{k}$ is contained in nondegenerate $k$-chains. 
We shall repeatedly use the following result, due to Iosevich, Sawyer, Taylor and Uriarte-Tuero:

Theorem 2.1 [Iosevich et al. 2014]. Let $T_{\lambda} f(x)=\lambda *(f \mu)(x)$, where $\lambda$ and $\mu$ are compactly supported, nonnegative Borel measures on $\mathbb{R}^{d}$. Suppose that $\mu$ satisfies (1-1) and, for some $\alpha>0$,

$$
|\hat{\lambda}(\xi)| \leq C|\xi|^{-\alpha} .
$$

Suppose that $v$ is a compactly supported Borel measure supported on $\mathbb{R}^{d}$ satisfying (1-1) with $s_{\mu}$ replaced by $s_{\nu}$ and suppose that $\alpha>d-s$, where $s=\frac{1}{2}\left(s_{\mu}+s_{\nu}\right)$. Then

$$
\left\|T_{\lambda} f\right\|_{L^{2}(v)} \leq c\|f\|_{L^{2}(\mu)} .
$$

In this article, we will use Theorem 2.1 with $\lambda=\sigma$, the surface measure on a $(d-1)$-dimensional sphere in $\mathbb{R}^{d}$. It is known — see [Stein 1993] — that

$$
\hat{\sigma}(\xi)=O\left(|\xi|^{-(d-1) / 2}\right) .
$$

Since the proof of Theorem 2.1 is short, we give the argument below for the sake of keeping the presentation as self-contained as possible. It is enough to show that

$$
\left\langle T_{\lambda \in} f, g v\right\rangle \leq C\|f\|_{L^{2}(\mu)} \cdot\|g\|_{L^{2}(v)} .
$$

The left-hand side equals

$$
\int \hat{\lambda}^{\epsilon}(\xi) \widehat{f \mu}(\xi) \widehat{g v}(\xi) d \xi
$$

By the assumptions of Theorem 2.1, the modulus of this quantity is bounded by

$$
C \int|\xi|^{-\alpha}|\widehat{f \mu}(\xi)||\widehat{g v}(\xi)| d \xi
$$

and applying Cauchy-Schwarz bounds this quantity by

$$
C\left(\int|\widehat{f \mu}(\xi)|^{2}|\xi|^{-\alpha_{\mu}} d \xi\right)^{\frac{1}{2}} \cdot\left(\int|\widehat{g v}(\xi)|^{2}|\xi|^{-\alpha_{\nu}} d \xi\right)^{\frac{1}{2}}
$$

for any $\alpha_{\mu}, \alpha_{v}>0$ such that $\alpha=\frac{1}{2}\left(\alpha_{\mu}+\alpha_{\nu}\right)$.

By Lemma 2.5 below, the quantity (2-2) is bounded by $C\|f\|_{L^{2}(\mu)} \cdot\|g\|_{L^{2}(v)}$ after choosing, as we may, $\alpha_{\mu}>d-s_{\mu}$ and $\alpha_{\nu}>d-s_{\nu}$. This completes the proof of Theorem 2.1.

Proof of Theorem 1.8 and Corollary 1.9. Let $\epsilon>0$. Divide both sides of (1-2) by $\epsilon^{k}$ and note that it suffices to establish the estimate

$$
C_{k}^{\epsilon}(\mu)=\int\left(\prod_{i=1}^{k} \sigma_{t_{i}}^{\epsilon}\left(x^{i+1}-x^{i}\right) d \mu\left(x^{i}\right)\right) d \mu\left(x^{k+1}\right) \leq c^{k},
$$

where $c$ is independent of $\epsilon$ and $t_{1}, \ldots, t_{k}>0$. Here $\sigma_{r}^{\epsilon}(x)=\sigma_{r} * \rho_{\epsilon}(x)$, with $\sigma_{r}$ the Lebesgue measure on the sphere of radius $r, \rho$ a smooth cut-off function with $\int \rho=1$ and $\rho_{\epsilon}(x)=\epsilon^{-d} \rho(x / \epsilon)$. Assume in addition that $\rho$ is nonnegative and that $\rho(x)=\rho(-x)$. 
Let $\sigma$ denote the Lebesgue measure on the $(d-1)$-dimensional sphere in $\mathbb{R}^{d}$. Set $T_{j}^{\epsilon}=T_{\sigma_{t_{j}}}^{\epsilon}$, where $T_{\sigma_{t_{j}}}^{\epsilon} f(x)=\sigma_{t_{j}} *(f \mu)(x)$ was introduced in Theorem 2.1. Define

$$
f_{k}^{\epsilon}(x)=T_{k}^{\epsilon} \circ \cdots \circ T_{1}^{\epsilon}(1)(x)
$$

and

$$
f_{0}^{\epsilon}(x)=1 .
$$

It is important to note that $f_{k}(x)$ depends implicitly on the choices of $t_{1}, \ldots, t_{k}>0$, and this choice will be made explicit throughout.

Observe that

$$
f_{k+1}^{\epsilon}=T_{k+1}^{\epsilon} f_{k}^{\epsilon} .
$$

Rewriting the left-hand side of (2-3), it suffices to show

$$
C_{k}^{\epsilon}(\mu)=\int f_{k}^{\epsilon}(x) d \mu(x) \leq c^{k} .
$$

Using Cauchy-Schwarz (and keeping in mind that $\int d \mu(x)=1$ ), we bound the left-hand side of (2-6) by

$$
C_{k}^{\epsilon}(\mu)=\int f_{k}^{\epsilon}(x) d \mu(x) \leq\left\|f_{k}^{\epsilon}\right\|_{L^{2}(\mu)} .
$$

We now use induction on $k$ to show that

$$
\left\|f_{k}^{\epsilon}\right\|_{L^{2}(\mu)} \leq c^{k},
$$

where $c$ is the constant obtained in Theorem 2.1. For the base case, $k=0$, we have $\left\|f_{0}^{\epsilon}\right\|_{L^{2}(\mu)}=\int d \mu(x)=1$. Next, we assume inductively that $\left\|f_{k}^{\epsilon}\right\|_{L^{2}(\mu)} \leq c^{k}$.

We now show that, for any $t_{k+1}>0$,

$$
\left\|f_{k+1}^{\epsilon}\right\|_{L^{2}(\mu)} \leq c^{k+1} .
$$

First, use (2-5) to write

$$
\left\|f_{k+1}^{\epsilon}\right\|_{L^{2}(\mu)}=\left\|T_{k+1}^{\epsilon} f_{k}^{\epsilon}\right\|_{L^{2}(\mu)} .
$$

Next, use Theorem 2.1 with $\lambda=\sigma$, the Lebesgue measure on the sphere, and $\alpha=\frac{1}{2}(d-1)$ (see the comment immediately following Theorem 2.1 to justify this choice of $\alpha$ ) to show that

$$
\left\|T_{k+1}^{\epsilon} f_{k}^{\epsilon}\right\|_{L^{2}(\mu)} \leq c\left\|f_{k}^{\epsilon}\right\|_{L^{2}(\mu)}
$$

whenever $s_{\mu}>d-\alpha=\frac{1}{2}(d+1)$.

We complete the proof by applying the inductive hypothesis. This completes the verification of (2-8).

We now recover Corollary 1.9. Let $s_{\mu} \in\left(\frac{1}{2}(d+1), \operatorname{dim}(E)\right)$, and choose a probability measure $\mu$ with support contained in $E$ which satisfies (1-1); the existence of such a measure is provided by Frostman's lemma (see [Falconer 1986], [Wolff 2003] or [Mattila 1995]). 
Cover $\Delta_{k}(E)$ with cubes of the form

$$
\bigcup_{i} \prod_{j=1}^{d}\left(t_{i j}, t_{i j}+\epsilon_{i}\right),
$$

where $\prod$ denotes the Cartesian product. We have $1=\mu \times \cdots \times \mu\left(E^{k+1}\right) \leq \sum_{i} \mu \times \cdots \times \mu\left\{\left(x^{1}, \ldots, x^{k+1}\right): t_{i j}-\epsilon \leq\left|x^{j+1}-x^{j}\right| \leq t_{i j}+\epsilon_{i}, 1 \leq j \leq k\right\}$.

By Theorem 1.8, the expression above is bounded by

$$
C \sum_{i} \epsilon_{i}^{k}
$$

and we conclude that (2-9) is bounded from below by $1 / C>0$. It follows that $\Delta_{k}(E)$ cannot have measure 0 and the proof of Corollary 1.9 is complete.

We now continue with the proof of Theorem 1.7.

Lower bound on $\boldsymbol{C}_{\boldsymbol{k}}^{\boldsymbol{\epsilon}}(\boldsymbol{\mu})$. Let $s_{\mu} \in\left(\frac{1}{2}(d+1), \operatorname{dim}(E)\right)$, and choose a probability measure $\mu$ with support contained in $E$ which satisfies (1-1).

We now establish the existence of a nonempty open interval $\tilde{I}$ such that

$$
\liminf _{\epsilon \rightarrow 0} C_{k}^{\epsilon}(\mu)>0
$$

where each $t_{i}$ belongs to $\tilde{I}$ and $C_{k}^{\epsilon}(\mu)$ is as in (2-3).

Note that this positive lower bound alone establishes the existence of vertices $x^{1}, \ldots, x^{k+1} \in E$ such that $\left|x^{i+1}-x^{i}\right|=t_{i}$ for each $i \in\{1, \ldots, k\}$ (this follows, for instance, by Cantor's intersection theorem and the compactness of the set $E$ ). Extra effort is made in the next section in order to guarantee that we may take $x^{1}, \ldots, x^{k+1}$ distinct.

We first prove the estimate (2-10) in the case that all gaps are equal. This is accomplished using a pigeonholing argument on chains of length one. We then provide a continuity argument to show that the estimate holds for variable gap values $t_{i}$ belonging to a nonempty open interval $\tilde{I}$. The second argument relies on the first precisely at the point when we show that the said continuous function is not identically equal to zero.

Lower bound for constant gaps. The proof of the estimate (2-10) in the case $k=1$ was already established in [Iosevich et al. 2012] provided that $\mu$ satisfies the ball condition in (1-1) with $\frac{1}{2}(d+1)<s_{\mu}<\operatorname{dim}_{\mathcal{H}}(E)$. The existence of such measures is established by Frostman's lemma (see, e.g., [Falconer 1986], [Wolff 2003] or [Mattila 1995]).

More specifically, it is demonstrated in [Iosevich et al. 2012] that there exists $c(1)>0, \epsilon_{0}>0$ and a nonempty open interval $I \subset(0$, diameter $(E))$ such that, if $t \in I$ and $0<\epsilon<\epsilon_{0}$, then

$$
C_{1}^{\epsilon}=\int \sigma_{t}^{\epsilon} * \mu(x) d \mu(x)>2 c(1) .
$$


To establish the estimate (2-10) for longer chains, we rely on the following lemmas:

Lemma 2.2. Set

$$
G_{t, \epsilon}(1)=\left\{x \in E: \sigma_{t}^{\epsilon} * \mu(x)>c(1)\right\} .
$$

There exists $m(1) \in \mathbb{Z}^{+}$such that, if $t \in I$ and $0<\epsilon<\epsilon_{0}$, then

$$
\mu\left(G_{t, \epsilon}(1)\right) \geq 2^{-2 m(1)} .
$$

Lemma 2.3. Set

$$
G_{t, \epsilon}(j+1)=\left\{x \in E:\left.\sigma_{t}^{\epsilon} * \mu\right|_{j}(x)>c(j+1)\right\},
$$

where $j \in\{1, \ldots,(k-1)\},\left.\mu\right|_{j}(x)$ denotes restriction of the measure $\mu$ to the set $G_{t, \epsilon}(j)$, and

$$
c(j+1)=\frac{1}{2} c(j) \mu\left(G_{t, \epsilon}(j)\right) .
$$

Then there exists $m(j+1) \in \mathbb{Z}^{+}$such that if $t \in I$ and $0<\epsilon<\epsilon_{0}$, then

$$
\mu\left(G_{t, \epsilon}(j+1)\right)>2^{-2 m(j+1)} .
$$

We postpone the proof of Lemmas 2.2 and 2.3 momentarily, and we apply these lemmas to obtain a lower bound on $C_{k}^{\epsilon}(\mu)$.

We write

$$
C_{k}^{\epsilon}(\mu)=\int f_{k}^{\epsilon}(x) d \mu(x),
$$

where $f_{k}^{\epsilon}$ was introduced in (2-4) and here $t_{1}=\cdots=t_{k}=t$.

Now

$$
C_{k}^{\epsilon}(\mu)=\int f_{k}^{\epsilon}(x) d \mu(x)=\iint \sigma_{t}^{\epsilon}(x-y) f_{k-1}(y) d \mu(y) d \mu(x) .
$$

Integrating in $x$ and restricting the variable $y$ to the set $G_{t, \epsilon}(1)$, we write

$$
C_{k}^{\epsilon}(\mu) \geq \int_{G_{t, \epsilon}(1)} \sigma_{t}^{\epsilon} * \mu(y) f_{k-1}(y) d \mu(y) \geq c(1) \int_{G_{t, \epsilon}(1)} f_{k-1}(y) d \mu(y)=c(1) \int f_{k-1}(y) d \mu_{1}(y) .
$$

To achieve a lower bound, we iterate this process. For each $j \in\{2, \ldots, k-1\}$ we have

$$
\begin{aligned}
\int f_{k-j}^{\epsilon}(x) d \mu_{j}(x)=\iint \sigma_{t}^{\epsilon}(x-y) f_{k-j-1}(y) d \mu(y) d \mu_{j}(x) & \geq \int_{G_{t, \epsilon}(j+1)} \sigma_{t}^{\epsilon} * \mu_{j}(y) f_{k-j-1}(y) d \mu(y) \\
& \geq c(j+1) \int_{G_{t, \epsilon}(j+1)} f_{k-j-1}(y) d \mu(y) \\
& =c(j+1) \int f_{k-j-1}(y) d \mu_{j+1}(y) .
\end{aligned}
$$

It follows that

$$
C_{k}^{\epsilon}(\mu) \geq\left(\prod_{j=1}^{k-1} c(i)\right) \iint \sigma_{t}^{\epsilon}(x-y) d \mu_{k-1}(y) d \mu(x) \geq\left(\prod_{j=1}^{k} c(i)\right) \mu\left(G_{t, \epsilon}(k)\right),
$$

and we are done in light of Lemma 2.3. 
Given Lemmas 2.2 and 2.3, we have shown that, for all $t \in I$ and all $0<\epsilon<\epsilon_{0}$, we have

$$
\liminf _{\epsilon \rightarrow 0} C_{k}^{\epsilon}(\mu)>0
$$

where all gap lengths $t_{1}, \ldots, t_{k}$ are constantly equal to $t$. This concludes the proof of the estimate (2-10) in the case of constant gaps.

We now proceed to the proofs of Lemmas 2.2 and 2.3.

Proof of Lemma 2.2. We write

$$
2 c(1)<\int \sigma_{t}^{\epsilon} * \mu(x) d \mu(x) \leq\left(\int_{\left(G_{t, \epsilon}(1)\right)^{c}} \sigma_{t}^{\epsilon} * \mu(x) d \mu(x)\right)+\left(\int_{G_{t, \epsilon}(1)} \sigma_{t}^{\epsilon} * \mu(x) d \mu(x)\right)=\mathcal{I}+\mathcal{I} \mathcal{I}
$$

where $A^{c}$ denotes the complement of a set $A \subset E$.

We first observe that

$$
\mathcal{I} \leq c(1)
$$

Next we estimate $\mathcal{I} \mathcal{I}$. Let $m \in \mathbb{Z}^{+}$and write

$$
G_{t, \epsilon}(1)=\left\{x \in E: c(1)<\sigma_{t}^{\epsilon} * \mu(x) \leq 2^{m}\right\} \cup\left\{x \in E: 2^{m} \leq \sigma_{t}^{\epsilon} * \mu(x)\right\} .
$$

Then

$$
\begin{aligned}
\mathcal{I I} & =\int_{\left\{x \in E: c(1)<\sigma_{t}^{\epsilon} * \mu(x) \leq 2^{m}\right\}} \sigma_{t}^{\epsilon} * \mu(x) d \mu(x)+\int_{\left\{x \in E: 2^{m} \leq \sigma_{t}^{\epsilon} * \mu(x)\right\}} \sigma_{t}^{\epsilon} * \mu(x) d \mu(x) \\
& \leq 2^{m} \mu\left(G_{t, \epsilon}(1)\right)+\sum_{l=m} 2^{l+1} \cdot \mu\left(\left\{x \in E: 2^{l} \leq \sigma_{t}^{\epsilon} * \mu(x) \leq 2^{l+1}\right\}\right) .
\end{aligned}
$$

We use Theorem 2.1 to estimate

$$
\mu\left(\left\{x \in E: 2^{l} \leq \sigma_{t}^{\epsilon} * \mu(x) \leq 2^{l+1}\right\}\right) \leq c_{d} \cdot 2^{-2 l},
$$

where the constant $c_{d}$ depends only on the ambient dimension $d$. Now,

$$
\mathcal{I I} \leq 2^{m} \mu\left(G_{t, \epsilon}(1)\right)+2 c_{d} \cdot \sum_{l=m} 2^{l} \cdot 2^{-2 l} \lesssim 2^{m} \mu\left(G_{t, \epsilon}(1)\right)+2^{-m} .
$$

It follows that

$$
2 c(1) \leq \mathcal{I}+\mathcal{I} \mathcal{I} \lesssim c(1)+2^{m} \mu\left(G_{t, \epsilon}(1)\right)+2^{-m} .
$$

Taking $m \in \mathbb{Z}^{+}$large enough, we conclude that

$$
\mu\left(G_{t, \epsilon}(1)\right) \geq 2^{-2 m} .
$$

Proof of Lemma 2.3. We prove the lemma by induction on $j$. The base case, $j=1$, was established in Lemma 2.2. Next, assume that there exists $m(j) \in \mathbb{Z}^{+}$such that

$$
2^{-m(j)}<\mu\left(G_{t, \epsilon}(j)\right)
$$

for all $0<\epsilon<\epsilon_{0}$ and $t \in I$. 
By the definition of $G_{t, \epsilon}(j)$,

$$
c(j) \mu\left(G_{t, \epsilon}(j)\right)<\left.\int_{G_{t, \epsilon}(j)} \sigma_{t}^{\epsilon} * \mu\right|_{G_{t, \epsilon}(j-1)}(x) d \mu(x) .
$$

Set $c(j+1)=\frac{1}{2} c(j) \mu\left(G_{t, \epsilon}(j)\right)$. By assumption, $2 c(j+1)=c(j) \mu\left(G_{t, \epsilon}(j)\right) \geq c(j) 2^{-m(j)}$, and in particular this quantity is positive. Next, we obtain a bound from above:

$$
\begin{aligned}
\left.\int_{G_{t, \epsilon}(j)} \sigma_{t}^{\epsilon} * \mu\right|_{G_{t, \epsilon}(j-1)}(x) d \mu(x) & \leq \int_{G_{t, \epsilon}(j)} \sigma_{t}^{\epsilon} * \mu(x) d \mu(x) \\
& =\left.\int \sigma_{t}^{\epsilon} * \mu\right|_{j}(x) d \mu(x) \\
& =\left(\left.\int_{\left(G_{t, \epsilon}(j+1)\right)^{c}} \sigma_{t}^{\epsilon} * \mu\right|_{j}(x) d \mu(x)\right)+\left(\left.\int_{G_{t, \epsilon}(j+1)} \sigma_{t}^{\epsilon} * \mu\right|_{j}(x) d \mu(x)\right) \\
& =\mathcal{I}+\mathcal{I} \mathcal{I} .
\end{aligned}
$$

First we observe that

$$
\mathcal{I} \leq c(j+1) .
$$

Next, we estimate $\mathcal{I} \mathcal{I}$. Let $m \in \mathbb{Z}^{+}$and write

$$
G_{t, \epsilon}(j+1)=\left\{x \in E: c(j+1)<\left.\sigma_{t}^{\epsilon} * \mu\right|_{j}(x) \leq 2^{m}\right\} \cup\left\{x \in E: 2^{m} \leq\left.\sigma_{t}^{\epsilon} * \mu\right|_{j}(x)\right\} .
$$

Then

$$
\begin{aligned}
\mathcal{I} \mathcal{I} & =\left.\int_{\left\{x \in E: c(j+1)<\left.\sigma_{t}^{\epsilon} * \mu\right|_{j}(x) \leq 2^{m}\right\}} \sigma_{t}^{\epsilon} * \mu\right|_{j}(x) d \mu(x)+\left.\int_{\left\{x \in E: 2^{m} \leq \sigma_{t}^{\epsilon} * \mu(x)\right\}} \sigma_{t}^{\epsilon} * \mu\right|_{j}(x) d \mu(x) \\
& \leq 2^{m} \cdot \mu\left(G_{t, \epsilon}(j+1)\right)+\sum_{l=m} 2^{l+1} \cdot \mu\left(\left\{x \in E: 2^{l} \leq\left.\sigma_{t}^{\epsilon} * \mu\right|_{j}(x) \leq 2^{l+1}\right\}\right) .
\end{aligned}
$$

We use Theorem 2.1 to estimate

$$
\mu\left(\left\{x \in E: 2^{l} \leq\left.\sigma_{t}^{\epsilon} * \mu\right|_{j}(x) \leq 2^{l+1}\right\}\right) \leq c_{d} \cdot 2^{-2 l},
$$

where the constant $c_{d}$ depends only on the ambient dimension $d$ and the choice of the measure $\mu$. Now,

$$
\mathcal{I I} \leq 2^{m} \mu\left(G_{t, \epsilon}(j+1)\right)+2 c_{d} \cdot \sum_{l=m} 2^{l} \cdot 2^{-2 l} \lesssim 2^{m} \mu\left(G_{t, \epsilon}(j+1)\right)+2^{-m} .
$$

It follows that

$$
2 c(j+1) \leq \mathcal{I}+\mathcal{I} \mathcal{I} \lesssim c(j+1)+2^{m} \mu\left(G_{t, \epsilon}(j+1)\right)+2^{-m} .
$$

Taking $m \in \mathbb{Z}^{+}$large enough, we conclude that

$$
\mu\left(G_{t, \epsilon}(j+1)\right) \geq 2^{-2 m} .
$$


Lower bound for variable gaps. We now verify (2-10) in the case of variable gap lengths. In more detail, we show that, for all $k \in \mathbb{Z}^{+}$and for values of $t_{i}$ in a nonempty open interval $\tilde{I}$, we have

$$
\liminf _{\epsilon \rightarrow 0} \int f_{k}^{\epsilon}(x) d \mu(x)>0,
$$

where $f_{k}^{\epsilon}$ is as defined in (2-4) with $0<t_{1}, \ldots, t_{k} \in \tilde{I}$.

The following lemma captures the strategy of the proof and establishes (2-12).

Lemma 2.4. We have

$$
C_{k}^{\epsilon}(\mu)=\int f_{k}^{\epsilon}(x) d \mu(x)=M_{k}\left(t_{1}, \ldots, t_{k}\right)-\sum_{j=1}^{k} R_{k, j}^{\epsilon}\left(t_{1}, \ldots, t_{k}\right),
$$

where

$$
M_{k}\left(t_{1}, t_{2}, \ldots, t_{k}\right)=\int \hat{\sigma}_{t_{k}}(\xi) \widehat{f_{k-1} \mu}(-\xi) \hat{\mu}(\xi) d \xi
$$

is continuous and bounded below by a positive constant (independent of $\epsilon$ ) on $\tilde{I} \times \cdots \times \tilde{I}$ for a nonempty open interval $\tilde{I}$, and

$$
R_{k, j}^{\epsilon}\left(t_{1}, t_{2}, \ldots, t_{k}\right)=\int \hat{\sigma}\left(t_{j} \xi\right)(1-\hat{\rho}(\epsilon \xi)) \widehat{f_{j-1} \mu}(\xi) \widehat{g_{j+1}^{\epsilon} \mu}(-\xi) d \xi=\mathscr{O}\left(\epsilon^{\alpha(s-(d+1) / 2)}\right)
$$

for some $\alpha>0$.

In proving the lemma, we utilize the notation

$$
g_{j}^{\epsilon}(x)=T_{j}^{\epsilon} \circ \cdots \circ T_{k}^{\epsilon}(1)(x)
$$

and

$$
g_{k+1}(x)=1 .
$$

It is important to note that $g_{j}(x)$ depends implicitly on the choices of $t_{1}, \ldots, t_{k}>0$, and this choice will be made explicit throughout.

First, we demonstrate (2-13) with repeated use of Fourier inversion. We again employ a variant of the argument in [Iosevich et al. 2012]. Write

$$
\int f_{k}^{\epsilon}(x) d \mu(x)=\iint \sigma_{t_{1}}^{\epsilon}(x-y) g_{2}^{\epsilon}(y) d \mu(x) d \mu(y)=\iint\left(\sigma_{t_{1}} * \rho_{\epsilon}\right)(x-y) g_{2}^{\epsilon}(y) d \mu(x) d \mu(y) .
$$

Using Fourier inversion and properties of the Fourier transform, this is equal to

$$
\iiint e^{2 \pi i(x-y) \cdot \xi} \hat{\sigma}_{t_{1}}(\xi) \hat{\rho}_{\epsilon}(\xi) g_{2}^{\epsilon}(y) d \mu(x) d \mu(y) d \xi
$$


Simplifying further, we write

$$
\begin{aligned}
\int f_{k}^{\epsilon}(x) d \mu(x) & =\int \hat{\sigma}_{t_{1}}(\xi) \hat{\rho}(\epsilon \xi) \hat{\mu}(\xi) \widehat{g_{2}^{\epsilon} \mu}(-\xi) d \xi \\
& =\int \hat{\sigma}_{t_{1}}(\xi) \hat{\mu}(\xi) \widehat{g_{2}^{\epsilon} \mu}(-\xi) d \xi+\int \hat{\sigma}_{t_{1}}(\xi)(1-\hat{\rho}(\epsilon \xi)) \hat{\mu}(\xi) \widehat{g_{2}^{\epsilon} \mu}(-\xi) d \xi \\
& =\int \hat{\sigma}_{t_{1}}(\xi) \hat{\mu}(\xi) \widehat{g_{2}^{\epsilon} \mu}(-\xi) d \xi+R_{k, 1}^{\epsilon}\left(t_{1}, t_{2}, \ldots, t_{k}\right) .
\end{aligned}
$$

With repeated use of Fourier inversion, we get

$$
\begin{aligned}
\int f_{k}^{\epsilon}(x) d \mu(x) & =\int \hat{\sigma}_{t_{j}}(\xi) \cdot \widehat{f_{j-1} \mu}(-\xi) \cdot \widehat{g_{j+1}^{\epsilon} \mu}(\xi) d \xi+\sum_{l=1}^{j} R_{k, l}^{\epsilon}\left(t_{1}, t_{2}, \ldots, t_{k}\right) \\
& \vdots \\
& =\int \hat{\sigma}_{t_{k}}(\xi) \cdot \widehat{f_{k-1} \mu}(-\xi) \cdot \hat{\mu}(\xi) d \xi+\sum_{l=1}^{k} R_{k, l}^{\epsilon}\left(t_{1}, t_{2}, \ldots, t_{k}\right) \\
& =M_{k}\left(t_{1}, t_{2}, \ldots, t_{k}\right)+\sum_{l=1}^{k} R_{k, l}^{\epsilon}\left(t_{1}, t_{2}, \ldots, t_{k}\right) .
\end{aligned}
$$

We now prove that $M_{k}\left(t_{1}, t_{2}, \ldots, t_{k}\right)$ is continuous on any compact set away from $\left(t_{1}, \ldots, t_{k}\right)=\overrightarrow{0}$ and that

$$
R_{k, j}^{\epsilon}\left(t_{1}, \ldots, t_{k}\right)=\mathcal{O}\left(\epsilon^{\alpha(s-(d+1) / 2)}\right) .
$$

Once these are established, we observe that the lower bound on constant chains established in (2-11) combined with (2-18) implies that $M_{k}\left(t_{1}, \ldots, t_{k}\right)$ is positive when $t_{1}=\cdots=t_{k}=t$ for any given $t \in I$. Fixing any such $t \in I$, it will then follow by continuity that $M_{k}\left(t_{1}, \ldots, t_{k}\right)$ is bounded from below on $\tilde{I} \times \cdots \times \tilde{I}$, where $\tilde{I}$ is a nonempty interval.

We now use the dominated convergence theorem to verify the continuity of $M_{k}\left(t_{1}, \ldots, t_{k}\right)$ on any compact set away from $\left(t_{1}, \ldots, t_{k}\right)=\overrightarrow{0}$. Let $t_{1}, \ldots, t_{k}>0$. Using properties of the Fourier transform and recalling the definition of $f_{j}$ from (2-4) and $g_{j}$ from (2-16), we write

$$
M_{k}\left(t_{1}, t_{2}, \ldots, t_{k}\right)=\int \hat{\sigma}_{t_{j}}(\xi) \cdot \widehat{f_{j-1} \mu}(-\xi) \cdot \widehat{g_{j+1} \mu}(\xi) d \xi
$$

for any $j \in\{1, \ldots, k\}$.

Let $h_{1}, \ldots, h_{k} \in \mathbb{R}$ be such that $\left(h_{1}, \ldots, h_{k}\right) \downarrow 0$. Let

$$
\tilde{f}_{j}=T_{t_{j}+h_{j}} \circ \cdots \circ T_{t_{1}+h_{1}}(1) \quad \text { and } \quad \tilde{g}_{j}=T_{t_{j}+h_{j}} \circ \cdots \circ T_{t_{k}+h_{k}}(1) .
$$

We have

$$
M_{k}\left(t_{1}+h_{1}, t_{2}+h_{2}, \ldots, t_{k}+h_{k}\right)=\int \hat{\sigma}_{t_{j}+h_{j}}(\xi) \cdot \widehat{\tilde{f}_{j-1} \mu}(-\xi) \cdot \widehat{\tilde{g}_{j+1} \mu}(\xi) d \xi
$$


The integrand goes to 0 as $h_{j}$ goes to 0 . Now, for $t_{j}$ in a compact set, the expression above is bounded by

$$
C\left(t_{j}\right) \int|\xi|^{-(d-1) / 2}\left|\widehat{\tilde{f}_{j-1} \mu}(-\xi)\right|\left|\widehat{\tilde{g}_{j+1} \mu}(\xi)\right| d \xi
$$

To proceed, we will utilize the following calculation:

Lemma 2.5. Let $\mu$ be a compactly supported Borel measure such that $\mu(B(x, r)) \leq C r^{s}$ for some $s \in(0, d)$. Suppose that $\alpha>d-s$. Then, for $f \in L^{2}(\mu)$,

$$
\int|\widehat{f \mu}(\xi)|^{2}|\xi|^{-\alpha} d \xi \leq C^{\prime}\|f\|_{L^{2}(\mu)}^{2} .
$$

To prove Lemma 2.5, observe that

$$
\int|\widehat{f \mu}(\xi)|^{2}|\xi|^{-\alpha} d \xi=C \iint f(x) f(y)|x-y|^{-d+\alpha} d \mu(x) d \mu(y)=\langle T f, f\rangle,
$$

where

$$
T f(x)=\int|x-y|^{-d+\alpha} f(y) d \mu(y)
$$

and the inner product above is with respect to $L^{2}(\mu)$. The positive constant $C$ appearing in (2-20) depends only on the ambient dimension $d$. Observe that

$$
\int|x-y|^{-d+\alpha} d \mu(y) \approx \sum_{j>0} 2^{j(d-\alpha)} \int_{|x-y| \approx 2^{-j}} d \mu(y) \leq C \sum_{j>0} 2^{j(d-\alpha-s)} \leq C^{\prime}
$$

since $\alpha>d-s$.

By symmetry, $\int|x-y|^{-d+\alpha} d \mu(x) \leq C^{\prime}$. It follows by using Schur's test [1911] — see also Lemma 7.5 in [Wolff 2003] — that

$$
\|T f\|_{L^{2}(\mu)} \leq C^{\prime}\|f\|_{L^{2}(\mu)} .
$$

This implies the conclusion of Lemma 2.5 by applying the Cauchy-Schwarz inequality to (2-20). We note that Lemma 2.5 can also be recovered from the fractal Plancherel estimate due to R. Strichartz [1990]. See also Theorem 7.4 in [Wolff 2003], where a similar statement is proved by the same method as above.

We already established, using [Iosevich et al. 2014], that finite compositions of the operators $T_{l}$ applied to $L^{2}(\mu)$ functions are in $L_{2}(\mu)$. Using the Cauchy-Schwarz inequality and in light of Lemma 2.5, $M_{k}\left(t_{1}+h_{1}, t_{2}+h_{2}, \ldots, t_{k}+h_{k}\right)$ is bounded. We proceed by applying the dominated convergence theorem. We have

$$
\begin{aligned}
\lim _{h_{j} \downarrow 0} M_{k}\left(t_{1}+\right. & \left.h_{1}, t_{2}+h_{2}, \ldots, t_{k}+h_{k}\right) \\
& =\int \hat{\sigma}_{t_{j}}(\xi) \cdot \widehat{\tilde{g}_{j-1} \mu}(-\xi) \cdot \widehat{\tilde{f}_{j+1} \mu}(\xi) d \xi \\
& =\int \hat{\sigma}_{t_{j}}(\xi) \cdot\left(T_{t_{j-1}+h_{j-1}} \circ \cdots \circ T_{t_{1}+h_{1}}(1) \cdot \mu \hat{)}(-\xi) \cdot\left(T_{t_{j+1}+h_{j+1}} \circ \cdots \circ T_{t_{k}+h_{k}}(1) \cdot \mu\right) \hat{(\xi) d \xi .}\right.
\end{aligned}
$$

We then rewrite the procedure, isolating $\hat{\sigma}_{t_{j}}$ for each $j \in\{1, \ldots, k\}$, and repeat the process above a total of $k$ times. 
Bounding the remainder. Next, we wish to show that $\lim _{\epsilon \downarrow 0} R_{k}^{\epsilon}\left(t_{1}, \ldots, t_{k}\right)=0$. Fix $\epsilon>0$. Recall that $R_{k}^{\epsilon}\left(t_{1}, \ldots, t_{k}\right)$ is equal to

$$
\int(1-\hat{\rho}(\epsilon \xi)) \hat{\sigma}(t \xi) \hat{\mu}(\xi) \widehat{f_{k} \mu}(-\xi) d \xi
$$

We consider the integral over $|\xi|<(1 / \epsilon)^{\alpha}$ and the integral over $|\xi|>(1 / \epsilon)^{\alpha}$ separately, where $\alpha \in(0,1)$ will be determined. Assume that $s>\frac{1}{2}(d+1)$.

Lemma 2.6. Let $\rho: \mathbb{R}^{d} \rightarrow \mathbb{R}$ satisfy the following properties: $\rho \geq 0, \rho(x)=\rho(-x)$, the support of $\rho$ is contained in $\{x:|x|<c\}$, and $\int \rho=1$. Then

$$
0 \leq 1-\hat{\rho}(\xi) \leq 2 \pi c|\xi| .
$$

To prove Lemma 2.6, write

$$
\hat{\rho}(\xi)=\int \cos (2 \pi x \cdot \xi) \rho(x) d x .
$$

We observe that $\cos x+|x|>1$, and conclude that the lemma follows when $|x|<c$. It follows that

$$
\int_{|\xi|<(1 / \epsilon)^{\alpha}}|\hat{\rho}(\epsilon \xi)-1||\hat{\sigma}(t \xi)||\hat{\mu}(\xi)|\left|\widehat{f_{k} \mu}(-\xi)\right| d \xi \lesssim \epsilon^{1-\alpha} \int|\hat{\sigma}(t \xi)||\hat{\mu}(\xi)|\left|\widehat{f_{k} \mu}(-\xi)\right| d \xi \lesssim \epsilon^{1-\alpha},
$$

where the last line is justified in the estimation of $M_{k}(t)$ above.

It remains to estimate the quantity

$$
\int_{|\xi|>(1 / \epsilon)^{\alpha}}|\hat{\sigma}(t \xi)||\hat{\mu}(\xi)|\left|\widehat{f_{k} \mu}(-\xi)\right| d \xi .
$$

Proceeding as in the estimation of $M_{k}(t)$ above, we bound the integral above by

$$
C t^{-(d-1) / 2} \int_{|\xi|>(1 / \epsilon)^{\alpha}}|\xi|^{-(d-1) / 2}|\hat{\mu}(\xi)|\left|\widehat{f_{k} \mu}(-\xi)\right| d \xi
$$

and then use Cauchy-Schwarz to bound it further by

$$
C t^{-(d-1) / 2}\left(\int_{|\xi|>(1 / \epsilon)^{\alpha}}|\xi|^{-(d-1) / 2}|\hat{\mu}(\xi)|^{2} d \xi\right)^{\frac{1}{2}}\left(\int_{|\xi|>(1 / \epsilon)^{\alpha}}|\xi|^{-(d-1) / 2}\left|\widehat{f_{k} \mu}(\xi)\right|^{2} d \xi\right)^{\frac{1}{2}} .
$$

We have already shown that the second integral is finite. The first integral is bounded by

$$
\sum_{j>\alpha \log _{2}(1 / \epsilon)} 2^{-j(d-1) / 2} \int_{2^{j} \leq|\xi|<2^{j+1}}|\hat{\mu}(\xi)|^{2} d \xi .
$$

We may choose a smooth cut-off function $\psi$ such that the inner integral is bounded by

$$
\int|\hat{\mu}(\xi)|^{2} \hat{\psi}\left(2^{-j} \xi\right) d \xi
$$

By Fourier inversion, this integral is equal to

$$
2^{d j} \iint \psi\left(2^{j}(x-y)\right) d \mu(x) d \mu(y) \leq C 2^{j(d-s)} .
$$


Returning to the sum, we now have the estimate

$$
C \sum_{j>\alpha} 2^{-j(d-1) / 2} \cdot 2^{j(d-s)} \leq C \sum_{j>\alpha} \sum_{\log _{2}(1 / \epsilon)} 2^{j(d+1) / 2-s} .
$$

As long as $s>\frac{1}{2}(d+1)$, this is $\ll \epsilon^{\alpha(s-(d+1) / 2)}$. Thus $R_{k}^{\epsilon}\left(t_{1}, \ldots, t_{k}\right)$ tends to 0 with $\epsilon$ as long as $\operatorname{dim}_{\mathscr{H}}(E)>\frac{1}{2}(d+1)$.

In conclusion, we have

$$
\lim _{\epsilon \downarrow 0} \int\left(\prod_{j=1}^{k} \sigma_{t_{j}}^{\epsilon}\left(x^{i+1}-x^{i}\right) d \mu\left(x^{i}\right)\right) d \mu\left(x^{k+1}\right)>c_{k}>0
$$

for all $t_{j} \in \tilde{I}$.

To complete the proof of Theorem 1.7 , it remains to verify that $E$ contains a nondegenerate $k$-chain with prescribed gaps. This is the topic of the next section.

\section{Nondegeneracy}

An important issue we have not yet addressed is that the chains we have found may be degenerate. As an extreme example, consider the case where $t_{i}=1$ for all $i$. Then included in our chain count are chains which simply bounce back and forth between two different points. We now take steps to ensure that we can indeed find chains with distinct vertices.

We verified above that there exists a nonempty open interval $\tilde{I}$ such that

$$
\lim _{\epsilon \downarrow 0} \int\left(\prod_{j=1}^{k} \sigma_{t_{j}}^{\epsilon}\left(x^{i+1}-x^{i}\right) d \mu\left(x^{i}\right)\right) d \mu\left(x^{k+1}\right)
$$

is bounded above and below for $t_{1}, \ldots, t_{k} \in \tilde{I}$. The upper bound appears in (2-3) and the lower bound appears in (2-21).

From here onward, we fix $t_{1}, \ldots, t_{k} \in \tilde{I}$ and set $\vec{t}=\left(t_{1}, \ldots, t_{k}\right)$. We now define a nonnegative Borel measure on the set of $k$-chains with the gaps $\vec{t}$. Let $\Lambda_{\vec{t}}^{k}$ denote a nonnegative Borel measure, defined as

$$
\Lambda_{\vec{t}}^{k}(A)=\lim _{\epsilon \downarrow 0} \int_{A}\left(\prod_{j=1}^{k} \sigma_{t_{j}}^{\epsilon}\left(x^{i+1}-x^{i}\right) d \mu\left(x^{i}\right)\right) d \mu\left(x^{k+1}\right),
$$

where $A \subset E \times \cdots \times E$, the $(k+1)$-fold product of the set $E$.

It follows that $\Lambda_{\vec{t}}^{k}$ is a finite measure which is not identically zero:

$$
0<\Lambda_{\vec{t}}^{k}(E \times \cdots \times E) .
$$

The strategy we use to demonstrate the existence of nondegenerate $k$-chains in $E$ is as follows: We first show that $\Lambda_{\vec{t}}^{k}$ has support contained in the set of $k$-chains. This is accomplished by showing that the measure has support contained in all "approximate" $k$-chains. We then show that the measure of the set of degenerate chains is zero. It follows, since the $\Lambda_{\vec{t}}^{k}$-measure of the set of $k$-chains is positive and 
the $\Lambda_{\vec{t}}^{k}$-measure of the set of degenerate $k$-chains is zero, that the set of nondegenerate $k$-chains in $E$ is nonempty.

For each test entry $n \in \mathbb{Z}^{+}$, define the sets of $(1 / n)$-approximate $k$-chains and the set of exact $k$-chains as

$$
A_{n, k}=\left\{\left(x^{1}, \ldots, x^{k+1}\right) \in E \times \cdots \times E: t_{i}-\frac{1}{n} \leq\left|x^{i+1}-x^{i}\right| \leq t_{i}+\frac{1}{n} \text { for each } i=1, \ldots, k\right\}
$$

and

$$
A_{k}=\left\{\left(x^{1}, \ldots, x^{k+1}\right) \in E \times \cdots \times E:\left|x^{i+1}-x^{i}\right|=t_{i} \text { for each } i=1, \ldots, k\right\} .
$$

Observe that

$$
\bigcap_{n} A_{n, k}=A_{k}
$$

We now observe that the support of $\Lambda_{t}^{k}$ is contained in the set of all approximate chains. This follows immediately from the observation that

$$
\Lambda_{\vec{t}}^{k}\left(A_{n, k}^{c}\right)=0
$$

for each $n \in \mathbb{Z}^{+}$, where $A_{n, k}^{c}$ denotes the complement of the set $A_{n, k}$ in $E \times \cdots \times E$.

Next, we observe that the support of $\Lambda_{\vec{t}}^{k}$ is contained in the set of exact chains. Indeed, it follows from the previous equation that

$$
\Lambda_{\bar{t}}^{k}\left(\bigcup_{n} A_{n, k}^{c}\right) \leq \sum_{n} \Lambda_{\vec{t}}^{k}\left(A_{n, k}^{c}\right)=0
$$

Recalling (3-1), we conclude that

$$
0<\Lambda_{\vec{t}}^{k}(E \times \cdots \times E)=\Lambda_{\vec{t}}^{k}\left(\bigcup_{n} A_{n, k}^{c}\right)+\Lambda_{\vec{t}}^{k}\left(\bigcap_{n} A_{n, k}\right),
$$

and so

$$
\Lambda_{\vec{t}}^{k}\left(A_{k}\right)=\Lambda_{\vec{t}}^{k}\left(\bigcap_{n} A_{n, k}\right)>0 .
$$

Since $t_{1}, \ldots, t_{k} \in \tilde{I}$ were chosen arbitrarily, we have shown that $\Lambda_{\vec{t}}^{k}\left(A_{k}\right)>0$ whenever $\vec{t}=\left(t_{1}, \ldots, t_{k}\right)$ and $t_{i} \in \tilde{I}$.

We now verify that the set of degenerate chains has $\Lambda_{\vec{t}}^{k}$-measure zero.

Lemma 3.1. Let

$$
D_{k}=\left\{\left(x^{1}, \ldots, x^{k+1}\right) \in E \times \cdots \times E: x^{i}=x^{j} \text { for some } i \neq j\right\} .
$$

Then

$$
\Lambda_{\vec{t}}^{k}\left(D_{k}\right)=0
$$

To prove the lemma, we first investigate the quantity

$$
\int_{D_{k}}\left(\prod_{j=1}^{k} \sigma_{t_{j}}^{\epsilon}\left(x^{i+1}-x^{i}\right) d \mu\left(x^{i}\right)\right) d \mu\left(x^{k+1}\right) .
$$


By the definition of $D_{k}$, we can bound the quantity above by

$$
\sum_{1 \leq m<n \leq k+1} \int_{\left\{\left(x^{1}, \ldots, x^{k+1}\right): x^{m}=x^{n}\right\}}\left(\prod_{j=1}^{k} \sigma_{t_{j}}^{\epsilon}\left(x^{i+1}-x^{i}\right) d \mu\left(x^{i}\right)\right) d \mu\left(x^{k+1}\right) .
$$

We can rewrite the integral as

$$
\int_{\left(\mathbb{R}^{d}\right)^{k}} \int_{\left\{x: x=x^{m}\right\}}\left(\prod_{j=1}^{k} \sigma_{t_{j}}^{\epsilon}\left(x^{i+1}-x^{i}\right)\right) d \mu\left(x^{n}\right) d \mu\left(x^{1}\right) \cdots d \mu\left(x^{n-1}\right) d \mu\left(x^{n+1}\right) \cdots d \mu\left(x^{k+1}\right) .
$$

Since the inside integral is taken over a region of measure 0 , this whole integral must be 0 . This holds for every choice of $m$ and $n$, and thus the entire sum must be 0 . This completes the proof of the lemma.

In conclusion, we have shown that the set of exact $k$-chains has positive measure $-\Lambda_{\vec{t}}^{k}\left(A_{k}\right)>0-$ and that the set of degenerate chains has zero measure $-\Lambda_{\vec{t}}^{k}\left(D_{k}\right)=0$. It follows that $A_{k} \neq D_{k}$ and $A_{k} \neq \varnothing$. In other words, there exists a nonempty open interval $\tilde{I}$ and distinct elements $x^{1}, \ldots, x^{k+1} \in E$ such that $\left|x^{i+1}-x^{i}\right|=t^{i}$ for each $i \in\{1, \ldots, k\}$.

\section{Acknowledgements}

The authors wish to thank Shannon Iosevich for her help with the diagrams used in this paper. The authors also wish to thank Fedja Nazarov and Jonathan Pakianathan for helpful discussions related to the subject matter of this article.

\section{References}

[Bourgain 1986] J. Bourgain, "A Szemerédi type theorem for sets of positive density in $\mathbb{R}^{k}$ ", Israel J. Math. 54:3 (1986), 307-316. MR 853455 Zbl 0609.10043

[Chan et al. 2013] V. Chan, I. Łaba, and M. Pramanik, "Finite configurations in sparse sets", preprint, 2013. arXiv 1307.1174

[Erdog̃an 2005] M. B. Erdog̃an, "A bilinear Fourier extension theorem and applications to the distance set problem", Int. Math. Res. Not. 2005:23 (2005), 1411-1425. MR 2152236 Zbl 1129.42353

[Eswarathasan et al. 2011] S. Eswarathasan, A. Iosevich, and K. Taylor, "Fourier integral operators, fractal sets, and the regular value theorem", Adv. Math. 228:4 (2011), 2385-2402. MR 2836125 Zbl 1239.28004

[Falconer 1985] K. J. Falconer, "On the Hausdorff dimensions of distance sets", Mathematika 32:2 (1985), 206-212. MR 834490 Zbl 0605.28005

[Falconer 1986] K. J. Falconer, The geometry of fractal sets, Cambridge Tracts in Mathematics 85, Cambridge University Press, 1986. MR 867284 Zbl 0587.28004

[Furstenberg et al. 1990] H. Furstenberg, Y. Katznelson, and B. Weiss, "Ergodic theory and configurations in sets of positive density", pp. 184-198 in Mathematics of Ramsey theory, edited by J. Nešetřil and V. Rödl, Algorithms and Combinatorics 5, Springer, Berlin, 1990. MR 1083601 Zbl 0738.28013

[Iosevich et al. 2012] A. Iosevich, M. Mourgoglou, and K. Taylor, "On the Mattila-Sjölin theorem for distance sets", Ann. Acad. Sci. Fenn. Math. 37:2 (2012), 557-562. MR 2987085 Zbl 1279.28006

[Iosevich et al. 2014] A. Iosevich, E. Sawyer, K. Taylor, and I. Uriarte-Tuero, "Fractal analogs of classical convolution inequalities", preprint, 2014.

[Maga 2010] P. Maga, "Full dimensional sets without given patterns", Real Anal. Exchange 36:1 (2010), 79-90. MR 3016405 Zbl 1246.28005 
[Mattila 1995] P. Mattila, Geometry of sets and measures in Euclidean spaces: fractals and rectifiability, Cambridge Studies in Advanced Mathematics 44, Cambridge University Press, 1995. MR 1333890 Zbl 0819.28004

[Schur 1911] J. Schur, "Bemerkungen zur Theorie der beschränkten Bilinearformen mit unendlich vielen Veränderlichen”, $J$. Reine Angew. Math. 140 (1911), 1-28. MR 1580823 JFM 42.0367.01

[Stein 1993] E. M. Stein, Harmonic analysis: real-variable methods, orthogonality, and oscillatory integrals, Princeton Mathematical Series 43, Princeton University Press, 1993. MR 1232192 Zbl 0821.42001

[Strichartz 1990] R. S. Strichartz, "Fourier asymptotics of fractal measures", J. Funct. Anal. 89:1 (1990), 154-187. MR 1040961 Zbl 0693.28005

[Wolff 1999] T. H. Wolff, "Decay of circular means of Fourier transforms of measures", Int. Math. Res. Not. 1999:10 (1999), 547-567. MR 1692851 Zbl 0930.42006

[Wolff 2003] T. H. Wolff, Lectures on harmonic analysis, edited by I. Łaba and C. Shubin, University Lecture Series 29 , American Mathematical Society, Providence, RI, 2003. MR 2003254 Zbl 1041.42001

[Ziegler 2006] T. Ziegler, "Nilfactors of $\mathbb{R}^{m}$-actions and configurations in sets of positive upper density in $\mathbb{R}^{m}$ ", J. Anal. Math. 99 (2006), 249-266. MR 2279552 Zbl 1145.37005

Received 10 Sep 2014. Revised 23 Apr 2015. Accepted 11 Oct 2015.

Michael BenNETT: bennett@math.rochester.edu

Department of Mathematics, University of Rochester, 915 Ray P. Hylan Building, 145 Dunrovin Lane, Rochester, NY 14628, United States

ALEXANDER IOSEVICH: iosevich@math.rochester.edu

Department of Mathematics, University of Rochester, 915 Ray P. Hylan Building, 145 Dunrovin Lane, Rochester, NY 14628, United States

KRYSTAL TAYLOR: taylor.2952@osu.edu

Department of Mathematics, The Ohio State University, 231 W. 18th Ave., MW 706, Columbus, OH 43210, United States 


\title{
Analysis \& PDE
}

msp.org/apde

\section{EDITORS}

\author{
EDITOR-IN-CHIEF \\ Patrick Gérard \\ patrick.gerard@math.u-psud.fr \\ Université Paris Sud XI \\ Orsay, France
}

BOARD OF EDITORS

Nicolas Burq Université Paris-Sud 11, France nicolas.burq@math.u-psud.fr

Massimiliano Berti Scuola Intern. Sup. di Studi Avanzati, Italy berti@sissa.it

Sun-Yung Alice Chang Princeton University, USA chang@math.princeton.edu

Michael Christ University of California, Berkeley, USA mchrist@math.berkeley.edu

Charles Fefferman Princeton University, USA cf@math.princeton.edu

Ursula Hamenstaedt Universität Bonn, Germany ursula@math.uni-bonn.de

Vaughan Jones U.C. Berkeley \& Vanderbilt University vaughan.f.jones@vanderbilt.edu

Vadim Kaloshin University of Maryland, USA vadim.kaloshin@gmail.com

Herbert Koch Universität Bonn, Germany koch@math.uni-bonn.de

Izabella Laba University of British Columbia, Canada ilaba@math.ubc.ca

Gilles Lebeau Université de Nice Sophia Antipolis, France lebeau@unice.fr

László Lempert Purdue University, USA lempert@math.purdue.edu

Richard B. Melrose Massachussets Inst. of Tech., USA rbm@math.mit.edu

Frank Merle Université de Cergy-Pontoise, France Frank.Merle@u-cergy.fr

William Minicozzi II Johns Hopkins University, USA minicozz@math.jhu.edu

Clément Mouhot Cambridge University, UK c.mouhot@dpmms.cam.ac.uk
Werner Müller

Yuval Peres

Gilles Pisier

Tristan Rivière

Igor Rodnianski

Wilhelm Schlag

Sylvia Serfaty

Yum-Tong Siu

Terence Tao

Michael E. Taylor

Gunther Uhlmann

András Vasy

Dan Virgil Voiculescu

Steven Zelditch

Maciej Zworski
Universität Bonn, Germany mueller@math.uni-bonn.de

University of California, Berkeley, USA peres@stat.berkeley.edu

Texas A\&M University, and Paris 6 pisier@math.tamu.edu

ETH, Switzerland riviere@math.ethz.ch Princeton University, USA irod@math.princeton.edu University of Chicago, USA schlag@math.uchicago.edu New York University, USA serfaty@cims.nyu.edu Harvard University, USA siu@math.harvard.edu University of California, Los Angeles, USA tao@math.ucla.edu Univ. of North Carolina, Chapel Hill, USA met@math.unc.edu

University of Washington, USA gunther@math.washington.edu

Stanford University, USA andras@math.stanford.edu

University of California, Berkeley, USA dvv@math.berkeley.edu

Northwestern University, USA zelditch@math.northwestern.edu

University of California, Berkeley, USA zworski@math.berkeley.edu

PRODUCTION

production@msp.org

Silvio Levy, Scientific Editor

See inside back cover or msp.org/apde for submission instructions.

The subscription price for 2016 is US \$/year for the electronic version, and \$/year (+\$, if shipping outside the US) for print and electronic. Subscriptions, requests for back issues from the last three years and changes of subscribers address should be sent to MSP.

Analysis \& PDE (ISSN 1948-206X electronic, 2157-5045 printed) at Mathematical Sciences Publishers, 798 Evans Hall \#3840, c/o University of California, Berkeley, CA 94720-3840, is published continuously online. Periodical rate postage paid at Berkeley, CA 94704, and additional mailing offices.

APDE peer review and production are managed by EditFlow ${ }^{\circledR}$ from MSP.

PUBLISHED BY

- mathematical sciences publishers

nonprofit scientific publishing

http://msp.org/

(C) 2016 Mathematical Sciences Publishers 


\section{ANALYSIS \& PDE}

\section{Volume $9 \quad$ No. $3 \quad 2016$}

Local analytic regularity in the linearized Calderón problem

JOHANNES SJÖSTRAND and GUNTHER UHLMANN

Dispersive estimates for the Schrödinger operator on step-2 stratified Lie groups

Hajer Bahouri, Clotilde Fermanian-KAmmerer and Is ABelle Gallagher

Obstructions to the existence of limiting Carleman weights

Pablo Angulo-Ardoy, Daniel Faraco, Luis GuiJarro and Alberto Ruiz

Finite chains inside thin subsets of $\mathbb{R}^{d}$

Michael Bennett, Alexander Iosevich and Krystal Taylor

Advection-diffusion equations with density constraints

ALPÁR RICHÁRD MÉSZÁROS and FILIPPO SANTAMBROGIO

Asymptotic stability in energy space for dark solitons of the Landau-Lifshitz equation

YAKINE BAHRI

On the well-posedness of the generalized Korteweg-de Vries equation in scale-critical $\hat{L}^{r}$ space

SATOShi Masaki and JUN-ICHI SEgatA

Regularity for parabolic integro-differential equations with very irregular kernels

RUSSELL W. SCHWAB and LuIS SilvestRe 